\title{
Long-Term Effects of Intragastric Instillations of Silastic 386 Foam Elastomer
}




\section{DISCLAIMER}

This report was prepared as an account of work sponsored by an agency of the United States Government. Neither the United States Government nor any agency Thereof, nor any of their employees, makes any warranty, express or implied, or assumes any legal liability or responsibility for the accuracy, completeness, or usefulness of any information, apparatus, product, or process disclosed, or represents that its use would not infringe privately owned rights. Reference herein to any specific commercial product, process, or service by trade name, trademark, manufacturer, or otherwise does not necessarily constitute or imply its endorsement, recommendation, or favoring by the United States Government or any agency thereof. The views and opinions of authors expressed herein do not necessarily state or reflect those of the United States Government or any agency thereof. 


\section{DISCLAIMER}

Portions of this document may be illegible in electronic image products. Images are produced from the best available original document. 
Edited by Jill Warren

\section{Photocomposition by Kris Mathieson}

Assisted by Joni Powell

\section{DISCLAIMER}

This report was prepared as an account of work sponsored by an agency of the United States Government. Neither the United States Government nor any agency thereof, nor any of their employees, makes any warranty, express or implied, or assumes any legal liability or responsibility for the accuracy, completeness, or usefulness of any information, apparatus, product, or process disclosed, or represents that its use would not infringe privately owned rights. References herein to any specific commercial product, process, or service by trade name, trademark, manufacturer, or otherwise, does not necessarily constitute or imply its endorsement, recommendation, or favoring by the United States Government or any agency thereof. The views and opinions of authors expressed herein do not necessarily state or reflect those of the United States Government or any agency thereof. 


\section{Long-Term Effects \\ of Intragastric Instillations of Silastic 386 Foam Elastomer}

D. M. Smith

G. A. Drake

J. E. London

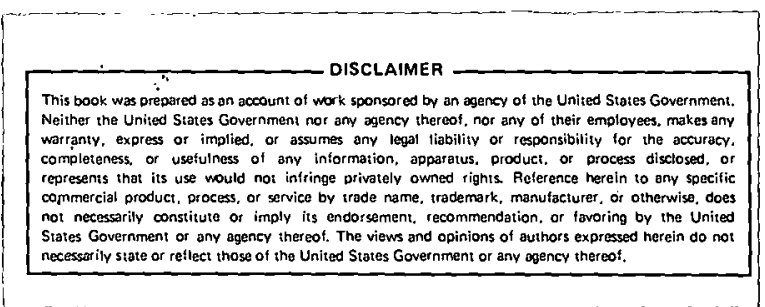




\title{
LONG-TERM EFFECTS OF INTRAGASTRIC INSTILLATIONS OF SILASTIC 386 FOAM ELASTOMER
}

by

D. M. Smith, G. A. Drake, and J. E. London

\begin{abstract}
Young male Sprague-Dawley rats ( 87 days old) were given a single dose [5 $\mathrm{g} / \mathrm{kg}$ body weight (BW)] of Silastic 386 foam elastomer intragastrically (IG), and young female Swiss-Webster mice ( 83 days old) were given a single dose (5 g/ $/ \mathrm{kg} \mathrm{BW}$ ) IG, or a dose (1 g/ $/ \mathrm{kg}$ BW) IG on each of 5 consecutive days, of Silastic 386 foam elastomer. All animals were then maintained for the rest of their lives and autopsied at death.

No significant effects resulting from the oral administration of this material were seen in any of the rats or mice.
\end{abstract}

\section{INTRODUCTION}

Previously, we reported the acute toxic properties for Silastic 386 foam elastomer.' Here, we present life-span data and frequency of degenerative and neoplastic diseases following a single intragastric (IG) instillation of Silastic 386 foam elastomer in rats and single and multiple IG instillations in mice.

\section{MATERIALS AND METHODS}

\section{A. Silastic 386 Foam Elastomer}

This material was supplied in $100-\mathrm{g}$ lots by Engineering Design Division, Los Alamos National Laboratory, Los Alamos, New Mexico. Before being used, it was stored in plastic bottles in our laboratory at $25^{\circ} \mathrm{C}$.

\section{B. Animals}

Young, male Sprague-Dawley (S-D) rats and young, female Swiss-Webster (S-W) mice were purchased from Charles River Laboratories, Wilmington, Massachusetts. Throughout the study, the mice were housed five per polycarbonate cage and the rats were housed two per cage. All were maintained on low-dust aspen-shaving bedding material, which was changed twice a week. The rats were fed Teklad ${ }^{\circledR}$ Rat Diet and the mice Teklad ${ }^{\circledR}$ Mouse Diet (Teklad Mills, Winfield, lowa). Chlorinated, acidified water was provided freely, and the animal holding rooms were maintained on a 12-hour-light, 12-hour-dark cycle.

\section{Instillation and Follow-up}

When they were 87 days old, the rats were fasted, sedated with ether, and given Silastic 386 
foam elastomer suspended in corn oil (Mazola, Best Foods, Englewood Cliffs, New Jersey) IG at a 5 $\mathrm{g} / \mathrm{kg}$ body weight (BW) dose. Sham controls and unmanipulated controls were included but are reported elsewhere. ${ }^{2}$

When the mice were 83 days old, they were divided randomly into two experimental groups. One group was given a single IG dose of Silastic 386 foam elastomer suspended in corn oil at the level of $5 \mathrm{~g} / \mathrm{kg} \mathrm{BW}$. The other group was given an IG dose of $1 \mathrm{~g} / \mathrm{kg}$ Silastic 386 foam elastomer in corn oil on each of 5 consecutive days. Controls were included but are reported elsewhere. ${ }^{2}$

All animals were then maintained in our animal colony for the rest of their lives. They were observed daily. Moribund animals were killed and autopsied immediately, and dead animals were autopsied as soon as possible.'Complete gross and microscopic post-mortem examinations were performed. All tissues were fixed in 10\% neutral acetate-buffered formalin, routinely processed, sectioned at $6 \mu \mathrm{m}$, and stained with hematoxylin-eosin.

\section{RESULTS AND DISCUSSION}

Life-span data for the experimental groups of rats and two groups of controls show no statistically significant differences among the three groups (Table I). No significant differences in tumor incidence were found in the experimental group compared to the two control groups (Table II). The incidence of tumors was similar to that reported in other studies. ${ }^{3.4}$

No significant differences in degenerative lesions were found in the experimental group com- pared to the two control groups (Table III). The incidence of degenerative diseases in the three groups was also consistent with those reported elsewhere..$^{4-6}$

Both experimental groups had longer median and mean life spans than the control group had, but the differences were not statistically significant (Table IV).

The incidence of tumors (except for malignant lymphoma) in the experimental groups was not significantly different from that in the controls (Table V), and is consistent with the reported incidence of neoplasms for S-W mice..$^{4-6}$

The elevated incidence of malignant lymphoma in female $S-W$ mice receiving five doses of the test material was statistically significant compared to controls, but this difference was not felt to be biologically significant because $31 \%$ of control female S-W mice "spontaneously" develop malignant lymphoma.* Degenerative lesions in the mice did not vary significantly in the experimental animals compared to the controls (Table VI) and were similar to those reported for S-W mice. ${ }^{4,6}$

\section{CONCLUSIONS}

The frequency of neoplasms and degenerative diseases in rats and mice was not affected by the administration of Silastic 386 foam elastomer. Furthermore, the life spans of the experimental rats and mice were not shortened compared to those in the control groups.

-M. W. Balk, Director of Laboratory Animal Medicine, Charles River Breeding Laboratory, Inc., Wilmington, Massachusetts, provided this information. 
TABLE I. Life Spans in Days $\left( \pm\right.$ SD $^{\mathrm{a}}$ ) for Rats

Single Dose Administered IG (5 g/kg BW)

\begin{tabular}{|c|c|c|c|}
\hline & $\begin{array}{l}\text { Experimental } \\
\text { Group }\left(7-3-18^{\circ}\right)\end{array}$ & $\begin{array}{c}\text { Sham } \\
\text { Control } \\
\text { Group }(7-3-8)\end{array}$ & $\begin{array}{l}\text { Unmanipulated } \\
\text { Control } \\
\text { Group (1-0-22) }\end{array}$ \\
\hline $\begin{array}{l}\text { Median } \\
\text { Mean } \\
\text { Standard error }\end{array}$ & $\begin{array}{c}893 \\
812 \pm 234 \\
(44)\end{array}$ & $\begin{array}{c}923 \\
890 \pm 147 \\
(41)\end{array}$ & $\begin{array}{c}830 \\
820 \pm 140 \\
(28)\end{array}$ \\
\hline
\end{tabular}

standard deviation.

Identification number.

TABLE II. Tumor Incidence in Rats

Single Dose Administered IG (5 g/kg BW)

\begin{tabular}{|c|c|c|c|}
\hline & $\begin{array}{l}\text { Experimental } \\
\text { Group }(7-3-18)\end{array}$ & $\begin{array}{c}\text { Sham } \\
\text { Control } \\
\text { Group }(7-3-8)\end{array}$ & $\begin{array}{l}\text { Unmanipulated } \\
\text { Control } \\
\text { Group (1-0-22) }\end{array}$ \\
\hline Malignant lymphoma & $2 / 21(10 \%)$ & $1 / 10(10 \%)$ & $2 / 19(11 \%)$ \\
\hline Fibrosarcoma & $3 / 21(14 \%)$ & $1 / 10(10 \%)$ & $0 / 19$ \\
\hline Leydig cell carcinoma & $1 / 21(5 \%)$ & $0 / 10$ & $0 / 19$ \\
\hline Islet cell tumors & $2 / 21(10 \%)$ & $1 / 10(10 \%)$ & $1 / 19(5 \%)$ \\
\hline Adrenal adenocarcinoma & $2 / 21(10 \%$ & $1 / 10(10 \%)$ & $3 / 19(16 \%)$ \\
\hline Pheochromocytoma & $1 / 21(5 \%)$ & $1 / 10(10 \%)$ & $0 / 19$ \\
\hline Lung adenocarcinoma & $1 / 21(5 \%)$ & $0 / 10$ & $0 / 19$ \\
\hline Myxosarcoma & $1 / 21(5 \%)$ & $0 / 10$ & $0 / 19$ \\
\hline \multirow{3}{*}{$\begin{array}{l}\text { Adrenal adenoma } \\
\text { Squamous cell carcinoma } \\
\text { of skin }\end{array}$} & $1 / 21(5 \%)$ & $0 / 10$ & $0 / 19$ \\
\hline & & & \\
\hline & $2 / 21(10 \%)$ & $0 / 10$ & $0 / 19$ \\
\hline
\end{tabular}

TABLE III. Degenerative Lesions in Rats

Single Dose Administered IG (5 g/kg BW)

\begin{tabular}{|c|c|c|c|}
\hline . & $\begin{array}{c}\text { Experimental } \\
\text { Group (7-3-18) }\end{array}$ & $\begin{array}{c}\text { Sham } \\
\text { Control } \\
\text { Group }(7-3-8)\end{array}$ & $\begin{array}{c}\text { Unmanipulated } \\
\text { Control } \\
\text { Group (1-0-22) }\end{array}$ \\
\hline $\begin{array}{l}\text { Glomerulonephrosis/ } \\
\text { glomerulonephritis } \\
\text { Reactive hyperplasia }\end{array}$ & $15 / 21(71 \%)$ & $6 / 10(60 \%)$ & $15 / 19(70 \%)$ \\
\hline of spleen & $5 / 21(29 \%)$ & $3 / 10(30 \%)$ & $2 / 19(11 \%)$ \\
\hline $\begin{array}{l}\text { Adrenal cortical hyperplasia } \\
\text { Liver-biliary hyperplasia }\end{array}$ & $\begin{array}{l}2 / 21(10 \%) \\
1 / 22(5 \%)\end{array}$ & $\begin{array}{l}0 / 10 \\
0 / 10\end{array}$ & $\begin{array}{l}0 / 19 \\
0 / 19\end{array}$ \\
\hline
\end{tabular}


TABLE IV. Life Spans in Days \pm SD (Mice)

\begin{tabular}{|c|c|c|c|}
\hline & $\begin{array}{c}\text { Single } \\
\text { Dose }^{\circ} \\
(7-3-14)\end{array}$ & $\begin{array}{c}\text { Five } \\
\text { Doses }^{b} \\
(7-3-25)\end{array}$ & $\begin{array}{l}\text { Control } \\
\text { Group } \\
(7-3-7)\end{array}$ \\
\hline $\begin{array}{l}\text { Median } \\
\text { Mean } \\
\text { Standard error }\end{array}$ & $\begin{array}{c}781 \\
767 \pm 178 \\
(34)\end{array}$ & $\begin{array}{c}861 \\
815 \pm 214 \\
(40)\end{array}$ & $\begin{array}{c}538 \\
568 \pm 129 \\
(36)\end{array}$ \\
\hline
\end{tabular}

${ }^{0} \mathbf{~ g / k g ~ B W ~ I G . ~}$

${ }^{\circ} \mathbf{~ g} / \mathbf{k g}$ BW IG.

TABLE V. Tumor Incidence (Mice)

\begin{tabular}{|c|c|c|c|}
\hline & $\begin{array}{c}\text { Single } \\
\text { Dose }^{\mathrm{a}} \\
(7-3-14)\end{array}$ & $\begin{array}{c}\text { Five } \\
\text { Doses }^{b} \\
(7-3-25)\end{array}$ & $\begin{array}{c}\text { Control } \\
\text { Group } \\
(7-3-7)\end{array}$ \\
\hline Lung adenoma & $0 / 11$ & $1 / 12(8 \%)$ & $3 / 13(23 \%)$ \\
\hline Lung adenocarcinoma & $2 / 11(18 \%)$ & $1 / 12(8 \%)$ & $1 / 13(8 \%)$ \\
\hline Osteosarcoma & $1 / 11(9 \%)$ & $0 / 12$ & $0 / 13$ \\
\hline Fibrosarcoma of subcutis & $2 / 11(18 \%)$ & $3 / 12(14 \%)$ & $0 / 13$ \\
\hline Reticulum cell sarcoma & $1 / 11(9 \%)$ & $1 / 12(8 \%)$ & $0 / 13$ \\
\hline Uterus adenocarcinoma & $2 / 11(18 \%)$ & $0 / 12$ & $0 / 13$ \\
\hline Malignant lymphoma & $0 / 11$ & $4 / 12(33 \%)^{c}$ & $0 / 13$ \\
\hline Basal cell carcinoma of skin & $0 / 11$ & $1 / 12(8 \%)$ & $0 / 13$ \\
\hline
\end{tabular}

${ }^{8} 5 \mathrm{~g} / \mathrm{kg}$ BW IG.

${ }^{\circ} \mathbf{~ g} / \mathbf{k g}$ BW IG.

i $P<0.025$ compared to control group 7-3-7.

TABLE VI. Degenerative Lesions (Mice)

Nephrosis

Spleen Iymphocytic hyperplasia

Pancreas nodular hyperplasia

Parathyroid hyperplasia

\begin{tabular}{|c|c|c|}
\hline $\begin{array}{c}\text { Single } \\
\text { Dose }^{a} \\
(7-3-14)\end{array}$ & $\begin{array}{c}\text { Five } \\
\text { Doses }^{b} \\
(7-3-25)\end{array}$ & $\begin{array}{c}\text { Control } \\
\text { Group } \\
(7-3-7)\end{array}$ \\
\hline $1 / 11(99 \%)$ & $1 / 12(8 \%)$ & $4 / 13(31 \%)$ \\
\hline $2 / 11(18 \%)$ & $3 / 12(25 \%)$ & $3 / 13(23 \%)$ \\
\hline $1 / 11(9 \%)$ & $.0 / 12$ & $0 / 13$ \\
\hline $0 / 11$ & $1 / 12(8 \%)$ & $0 / 13$ \\
\hline
\end{tabular}

${ }^{a} 5 \mathrm{~g} / \mathrm{kg}$ BW IG.

${ }^{1} \mathbf{g} / \mathbf{k g} B W$ IG. 


\section{REFERENCES}

1. D. M. Smith, J. E. London, G. A. Drake, and R. G. Thomas, "A Preliminary Toxicological Study of Silastic 386 Foam Elastomer," Los Alamos Scientific Laboratory report LA-7365-MS (June 1978).

2. D. M. Smith, G. A. Drake, and J. E. London, "Long-Term Effects of Intragastric Instillations of BDNPF:BDNPA in Male Sprague-Dawley Rats and Female Swiss-Webster Mice," Los Alamos National Laboratory report LA-8924-MS (July 1981).

3. D. G. Goodman, N. H. Ahman, H. Bazin, G. Burtonboy, A. Bectieve, and R. W. Baldwin, "Tumors: Rat. Part I. Spontaneous," in Inbred and Genetically Defined Strains of Laboratory Animals, Part I. Mouse and Rat, P. O. Altman and D. D. Katz, Eds. (Federation of American Societies for Experimental Biology, Bethesda, Maryland, 1979), pp. 298-301.

4. K. Benirschke, Pathology of Laboratory Animals, Vols. I and II, F. M. Garner and J. C. Jones, Eds. (Springer-Verlag, New York, 1978).

5. M. W. Balk, Director of Laboratory Animal Medicine, letter regarding disease incidences in Sprague-Dawley rats maintained in the Charles River Breeding Laboratory, Inc., Wilmington, Massachusetts, to David M. Smith, LS-1, Los Alamos Nátional Labortory (May 15, 1981).

6. P. L. Altman and D. D. Katz, Inbred and Genetically Defined Strains of Laboratory Animals, Part 1. Mouse and Rat (Federation of American Societies for Experimental Biology, Bethesda, Maryland, 1979). 


\begin{tabular}{|c|c|c|c|c|c|c|c|c|c|c|c|}
\hline & & & & $\begin{array}{l}\text { Pri } \\
\mathrm{Na}\end{array}$ & $\begin{array}{l}\text { ed in the Unit } \\
\text { Availa } \\
\text { onal Technical } \\
\text { US Departme } \\
5285 \text { Port } \\
\text { Springfield }\end{array}$ & $\begin{array}{l}\text { States of Am } \\
\text { e from } \\
\text { nformation Se } \\
\text { of Commerce } \\
\text { oyal Road } \\
\text { VA } 22161 \\
\text { Sn }\end{array}$ & & & & & \\
\hline Page Range & $\begin{array}{c}\text { Domestic } \\
\text { Price }\end{array}$ & $\begin{array}{c}\text { NTIS } \\
\text { Price Code } \\
\end{array}$ & Page Range & $\begin{array}{l}\text { Domestic } \\
\text { Price }\end{array}$ & $\begin{array}{c}\text { NTIS } \\
\text { Price Code }\end{array}$ & Page Range & $\begin{array}{l}\text { Domestic } \\
\text { Price }\end{array}$ & $\begin{array}{c}\text { NTIS } \\
\text { Price Code }\end{array}$ & Page Range & $\begin{array}{c}\text { Domestic } \\
\text { Price }\end{array}$ & $\begin{array}{c}\text { NTIS } \\
\text { Price Code }\end{array}$ \\
\hline $001-025$ & 55.00 & $\mathrm{~A} 02$ & $151-175$ & $\$ 11.00$ & A08 & $301-325$ & $\$ 17.00$ & A 14 & $451-475$ & $\$ 23.00$ & $\mathrm{~A} 20$ \\
\hline $026-050$ & 6.00 & $\mathrm{~A} 03$ & $176-200$ & 12.00 & $\mathrm{~A} 09$ & 326.350 & $18 \cap 0$ & A 15 & $476-500$ & 24.00 & A 21 \\
\hline $051-075$ & 7.00 & $\mathrm{~A} 04$ & $201-225$ & 13.00 & A 10 & $351-375$ & 19.00 & A 16 & $501-525$ & 25.00 & A 22 \\
\hline $076-100$ & 8.00 & A05 & $226-250$ & 14.00 & Al1 & $376-400$ & 20.00 & $A 17$ & $526-550$ & 26.00 & $\mathrm{~A} 23$ \\
\hline $101-125$ & 9.00 & A06 & $251-275$ & 15.00 & A 12 & $401-425$ & 21.00 & A 18 & 551.575 & 27.00 & A24 \\
\hline $126-150$ & 10.00 & $\mathrm{~A} 07$ & $276-300$ & 16.00 & A 13 & $426-450$ & 22.00 & A 19 & $\begin{array}{c}576-600 \\
601-u p\end{array}$ & $\begin{array}{c}28.00 \\
+\end{array}$ & $\begin{array}{l}\text { A } 25 \\
\text { A } 99\end{array}$ \\
\hline
\end{tabular}

†Add $\$ 1.00$ for each additional 25-page increment or portion thereof from 601 pages up. 
Los Alaños 